\title{
O PROJETO DE EXTENSÃO "CAPACITAÇÃO DE TUTORIA EM NATAÇÃO ADAPTADA”
}

\author{
THE EXTENSION PROJECT "TRAINING OF TUTORING IN \\ ADAPTED SWIMMING"
}

\author{
Juliana Aparecida de Paula Schuller* \\ Lilian Cristina Gomes do Nascimento** \\ Paulo de Tarso Nazar*** \\ Cléria Maria Lobo Bittar**** \\ Maria Georgina Marques Tonello*****
}

Resumo: Este trabalho tem como objetivo apresentar o projeto de extensão "Capacitação de tutoria em natação adaptada". A ação foi promovida para contribuir com a formação universitária dos acadêmicos, sendo dividida em conteúdo teórico, conteúdo prático e intervenção como tutores de nadadores com deficiência física. Participaram do módulo teórico da capacitação 32 acadêmicos, dos quais 9 atuaram como tutores. Destes, 5 informaram ter conhecidos ou familiares com deficiência e 4 declararam não ter nenhum contato anterior com essa população. Nenhum tinha experiência prévia em atuar como tutor de pessoas com deficiência. Para os graduandos que participaram da ação de tutoria, observou-se que eles demonstraram maior interesse em atuar com pessoas com deficiência como foco de atuação após formados. Conclui-se que a participação no projeto de extensão auxiliou no processo de formação profissional dos participantes, além de contribuir para uma mudança positiva sobre a percepção da pessoa com deficiência.

Palavras-chave: Extensão; Pessoas com deficiência; Tutoria.

\begin{abstract}
This study aims to present the university extension project called "Training of tutoring in adapted swimming". This action was promoted to contribute to the academic education of the students, being divided into theoretical content, practical content and intervention as tutors of swimmers with physical disabilities. Thirty-two undergraduate students participated in the theoretical training module, of which 09 worked as tutors. Five of the tutors reported having acquaintances or relatives with disabilities and 04 reported having no previous contact with this population. None of them had previous experience in working as tutors for people with disabilities. The undergraduates who participated in the tutorial action were seen to show greater interest in working with people with disabilities as a focus of action after training than those who did not take part in the intervention. The results led to the conclusion that the participation in the extension project helped in the process of professional training of the participants, besides contributing to a positive change in their perception of disabled people.
\end{abstract}

Keywords: Extension; Disabled people; Peer tutoring.

\footnotetext{
* Professora da Universidade Federal de Mato Grosso (UFMT), Cuiabá, MT - Brasil. Aluna de Doutorado da Universidade de Franca (UNIFRAN), Franca, SP - Brasil. E-mail: juliana_schuller@yahoo.com.br

** Professora da Universidade de Franca (UNIFRAN), Franca, SP - Brasil. E-mail: liliangomes@hotmail.com.br *** Professor da Universidade de Franca (UNIFRAN), Franca, SP - Brasil. E-mail: paulo.nazar@unifran.edu.br **** Professora da Universidade de Franca (UNIFRAN), Franca, SP - Brasil. E-mail: cleria.bittar@unifran.edu.br ***** Professora da Universidade de Franca (UNIFRAN), Franca, SP - Brasil. E-mail: gina@ginatonello.com.br
} 


\section{Introdução}

A função de uma universidade está pautada na tríade ensino, pesquisa e extensão, e conforme Foletto Pivetta et al. (2010), estas atividades, complementares e interdependentes, devem ter o mesmo valor no sistema universitário, e quando isto não acontece, arrisca-se na obtenção do desenvolvimento de conhecimento mutilante e reducionista, uma vez que a qualidade dos profissionais formados depende, em grande parte, do nível de interação e articulação entre estes três pilares do conhecimento.

A extensão universitária trata-se de um dos modos de interação entre a universidade e a comunidade na qual está inserida, em que a instituição acadêmica leva os conhecimentos e/ou assistência aos diferentes setores da sociedade e tem como retorno as respostas aos questionamentos dos programas propostos, além de aprender com o saber das comunidades participantes (NUNES; DA CRUZ SILVA, 2011). Trata-se de um dos caminhos para desenvolver uma formação acadêmica completa, integrando teoria e prática, num processo de comunicação com a sociedade, propiciando a troca de conhecimento entre (MANCHUR; SURIANI; CUNHA, 2013).

Segundo Arroyo e Rocha (2010), a implementação das ações de extensão exigem que as universidades que as propõem assumam uma concepção de valorização das ações junto à comunidade,e se estendem à sociedade como um todo.

Entendendo o papel dos projetos de extensão como primordiais no estreitamento do vínculo com a comunidade na qual estão inseridos, não podemos desconsiderar as atividades voltadas às parcelas populacionais consideradas como "minorias" e que podem estar em situação de vulnerabilidade em função de sua condição as pessoas com deficiências.

\section{Pessoas com deficiência são}

[...] aquelas que têm impedimentos de longo prazo de natureza física, mental, intelectual ou sensorial, os quais, em interação com diversas barreiras, podem obstruir sua participação plena e efetiva na sociedade em igualdades de condições com as demais pessoas (BRASIL, 2009).

Segundo a OMS, os indivíduos com deficiência apresentam desvantagem como consequência de sua incapacidade, o que ocasiona uma limitação no desempenho de um papel social que é o considerado normal (WHO,1980).

Para o paradigma médico, a deficiência é vista como "uma condição da pessoa, resultante de um impedimento, limitação, perda ou anormalidade numa parte do corpo ou numa função". Neste sentido, a diferença representa aspecto negativo do funcionamento, portanto uma doença ou defeito, já que existe a comparação com o idealizado, "quanto mais próximo do modelo, mais perfeito; quanto mais distante, mais imperfeito" (ABENHAIM, 2009, p.
237). Para Omote (1994), as diferenças individuais são as características especificas apresentadas pelas pessoas, mas, à medida que algumas diferenças se destacam e a elas são atribuídas significações de descrédito, ou ainda, de desvantagem, essas diferenças não representam mais variações nas características inerentes às pessoas.

Já o paradigma social reconhece as dificuldades que algumas pessoas têm ao realizar determinadas tarefas, mas considera como fator limitador a condição imposta pelo contexto social ao criar barreiras físicas, programáticas e atitudinais, e neste ponto de vista, a diferença é, portanto, um desafio a ser superado pela sociedade, em que cada pessoa "é reconhecida como ser único e irrepetível com direito a desenvolver sua potencialidade" (ABENHAIM, 2009, p. 237).

Neste sentido, as diferenças resultantes da deficiência não são marcadas como diferenças individuais e podem ser percebidas como limitações inerentes àqueles que as possuem, uma marca, um estereótipo, mesmo nos dias atuais, quando tanto falamos sobre a necessidade de se aceitarem as diferenças e buscamos a inserção dos "diferentes" no contexto social. Precisa-se entender que os que diferem não necessariamente são as minorias, mas talvez aqueles que mais sofrem com as questões relacionadas à vulnerabilidade social, já que, conforme dados da Organização Mundial de Saúde da estimativa de 2010, cerca de $15 \%$ da população mundial possui algum tipo de deficiência (WHO, 2012), e no Brasil, conforme estimativa do mesmo ano, $23,90 \%$ de brasileiros apresenta alguma deficiência (IBGE, 2012).

Aliado a possíveis condições de vulnerabilidade da pessoa com deficiência, podemos encontrar o sedentarismo, uma das principais preocupações das agências de saúde na atualidade. Esta situação se dá em função da histórica falta de oportunidades de participação nas mais diversas atividades sociais, incluindo a prática de atividade física, o que torna mais agravantes as consequências do sedentarismo nesta população.

Tendo em vista os aspectos apresentados, o objetivo deste trabalho é apresentar o projeto de extensão "Capacitação de Tutoria em Natação Adaptada", desenvolvido a fim de capacitar acadêmicos para atuarem com pessoas com deficiência física.

\section{Método}

$\mathrm{O}$ presente estudo foi desenvolvido em uma universidade particular localizada em um município no nordeste do estado de São Paulo, Brasil, tendo sido aprovado pelo Comitê de Ética sob protocolo no. 41976915.0.0000.5495, conforme Resolução 466/12 do Conselho Nacional de Saúde. 
Inicialmente, foi oferecido gratuitamente a todos os alunos regularmente matriculados nos cursos licenciatura e bacharelado em educação física da instituição de realização da pesquisa, na modalidade de extensão universitária, o curso de capacitação teórica intitulado: "Tutoria em Natação Adaptada Para Pessoas com Deficiência Física", dividido em conteúdo teórico e conteúdo prático.

\section{O Curso de Capacitação}

O curso de capacitação foi ministrado pelos pesquisadores envolvidos neste trabalho, por fins didáticos, e o conteúdo desenvolvido foi dividido em blocos temáticos de aprendizagem (Quadro 1).

Quadro 1. Programa de Treinamento da Capacitação em Tutoria
\begin{tabular}{|c|l|l|}
\hline $\begin{array}{c}\text { Carga } \\
\text { horária }\end{array}$ & \multicolumn{1}{|c|}{ Atividade } & \multicolumn{1}{c|}{ Conteúdo } \\
\hline & $\begin{array}{l}\text { Apresentação do conteúdo } \\
\text { Aula teórica sobre introdução à } \\
\text { deficiência, pessoas com } \\
\text { necessidades especiais e tutoria }\end{array}$ & $\begin{array}{l}\text { - Adquirir conhecimentos básicos em } \\
\text { relação à deficiência fisica e à tutoria. } \\
\text { - Definição e conceitos de deficiência, } \\
\text { necessidades especiais e tutoria. }\end{array}$ \\
\hline 2 & $\begin{array}{l}\text { Aprendizagem motora: tipos } \\
\text { de instrução, técnicas de } \\
\text { motivação e reforço positivo. }\end{array}$ & $\begin{array}{l}\text { Política Nacional de Promoção da da princípios da aprendizagem e de } \\
\text { Saúde para pessoas com deficiência }\end{array}$ \\
\hline 2 & $\begin{array}{l}\text { Aula teórica sobre introdução à } \\
\text { natação }\end{array}$ & $\begin{array}{l}\text { Princípios básicos e estratégias do } \\
\text { estilo dos nados }\end{array}$ \\
\hline 2 & $\begin{array}{l}\text { Aula prática de natação } \\
\text { adaptada }\end{array}$ & $\begin{array}{l}\text { Princípios específicos e estratégias do } \\
\text { estilo dos nados e atuação prática }\end{array}$ \\
\hline
\end{tabular}

\section{A Prática de Tutoria em Natação Adaptada}

O projeto de tutoria em natação adaptada foi desenvolvido em uma piscina coberta e aquecida, da universidade participante (Figura 1).

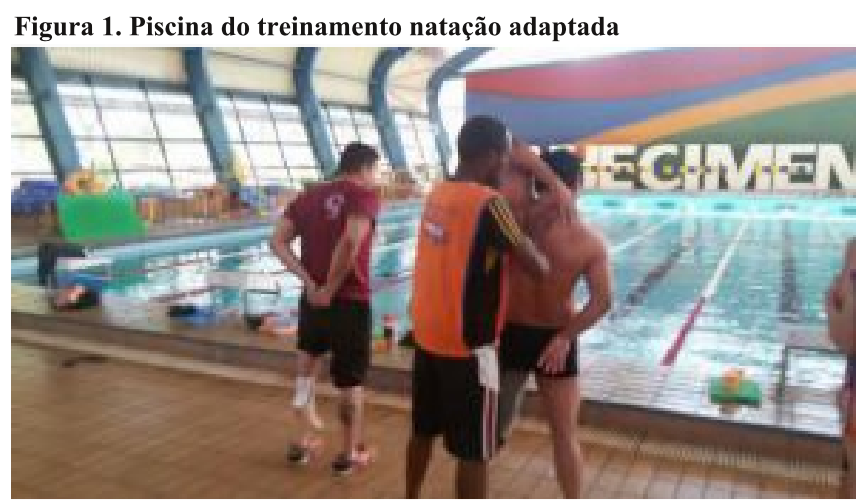

Fonte: Dados da pesquisa

Os critérios de inclusão nesta etapa foram: os estudantes que realizaram todo o curso teórico, atingida a meta de $100 \%$ de presença no curso de capacitação, apresentarem interesse e disponibilidade de tempo para participar como tutores dos atletas no projeto. Como critérios de exclusão para atuar como tutores foram recusados os alunos que tivessem atuado previamente como tutor de pessoas com deficiência.

Os treinamentos de natação para os 10 atletas com deficiência física foram desenvolvidos em colaboração pelo técnico responsável da equipe, o qual é profissional de educação física, tinha experiência prévia em treinamento de natação com atletas e aceitou colaborar com o projeto. A pesquisa foi conduzida em protocolo de 12 semanas, com treino dos atletas 4 vezes por semana (segunda a quinta-feira), sendo que os tutores foram divididos em 2 grupos para participação do projeto 2 vezes por semana.

Os tutores receberam orientações do técnico de natação adaptada no início do treinamento $\mathrm{e}$ desenvolveram as atividades de tutoria conforme programado. As atividades constavam de apoio, acompanhamento e correção dos atletas com deficiência física (Figura 2).

Figura 2. Interação dos acadêmicos como tutores com os atletas com deficiência

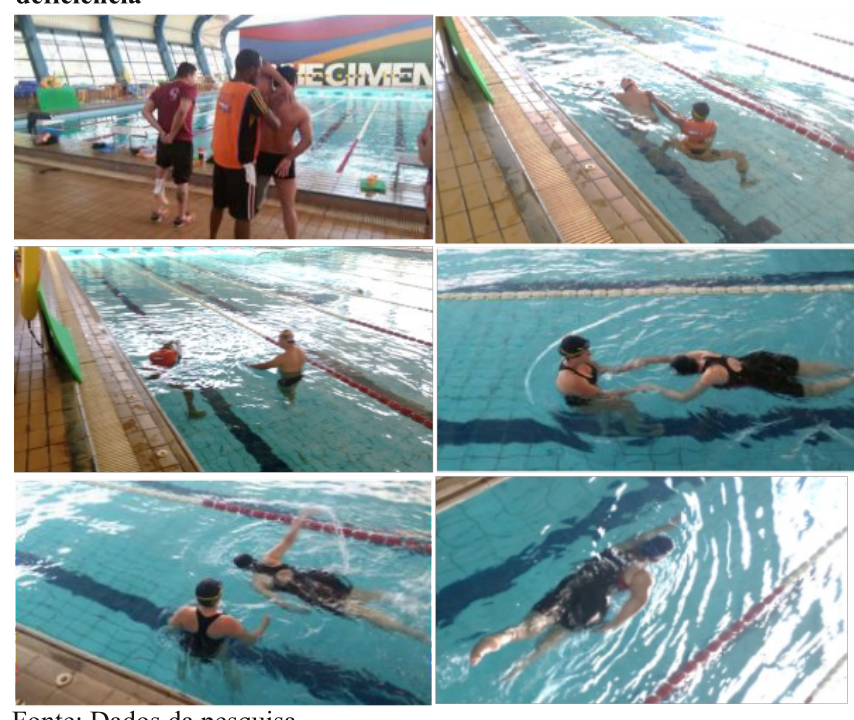

Fonte: Dados da pesquisa

Por conseguinte, este projeto de extensão foi desenvolvido buscando capacitar os acadêmicos participantes a atuar com nadadores com deficiência, utilizando-se, para tanto, da tutoria.

\section{Resultados}

Após a divulgação, obteve-se um total de 46 alunos interessados em participar do curso. Realizaram totalmente o módulo teórico do curso de capacitação 32 acadêmicos.

Após o término do curso teórico, os participantes foram convidados a participar do projeto: "Treinamento em natação adaptada para pessoas com deficiências físicas", para atuarem como tutores na intervenção prática com os atletas. 
Os envolvidos estavam orientados pelo princípio da indissociabilidade entre ensino, pesquisa e extensão (ZANUTTO; ISHIDA; DUARTE, 2017). O projeto apresenta uma estrutura organizacional composta por três módulos (Figura 3).

Figura 3. Estrutura organizacional do projeto.

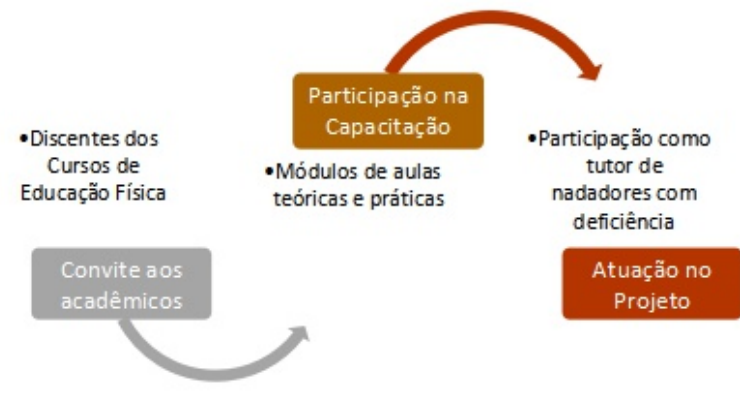

Figura 3. Estrutura organizacional do projeto.

Participaram como tutores um total de 9 acadêmicos, sendo 6 do sexo masculino e 3 do sexo feminino, com uma média etária de 26,4 $\pm 4,06$ anos. Cada tutor realizou o acompanhamento de um atleta por aula, ocorrendo um rodízio desse acompanhamento, para que os tutores pudessem vivenciar as mais diferentes situações no ambiente ensino-aprendizagem. Dos 9 participantes que atuaram como tutores, nenhum apresentava algum tipo de deficiência, ao passo que 5 informaram ter conhecidos ou familiares com deficiência e 4 declararam não ter nenhum contato anterior com essa população.

Ao considerar o ensino superior, entende-se que as universidades ocupam um papel primordial na sociedade, podendo contribuir de modo efetivo para acarretar mudanças favoráveis à sociedade. Segundo Sarmiento (2017), por sua posição privilegiada no contexto social, as universidades, através das práticas de promoção da saúde e do bem-estar, podem contribuir positivamente para a saúde e educação da sociedade em geral, além dos estudantes e funcionários, através de suas políticas e práticas ofertadas.

Se o ensino repousa sobre o "já conhecido", a pesquisa se dirige ao "ainda não conhecido". Buscase, pois, transformar o "ainda não conhecido" em algo conhecido; daí a tendência a se considerar que o ensino decorre da pesquisa: só pode haver ensino a respeito das coisas que se conhecem, que foram aprendidas. Todavia, só se pode aprender se houver conhecimentos sistematizados e a função da pesquisa é justamente produzir esses conhecimentos. Assim, na medida em que esses conhecimentos são produzidos, é possível difundi-los, ensiná-los a outras pessoas; daí resulta a necessidade de articular ensino e pesquisa às atividades extensionistas no âmbito das universidades (DOS SANTOS, 2012, p. 157)

Podemos observar que o projeto propiciou aos acadêmicos participantes um maior conhecimento acerca das vicissitudes referentes às pessoas com deficiência física; ademais, a prática diária com os atletas acarretou numa dissociação da imagem da pessoa com deficiência associada somente à condição de limitação, transformando a visão dos acadêmicos no sentido de vislumbrar, a partir deste convívio, as potencialidades das pessoas com deficiência.

A participação em projetos de extensão nas universidades trata-se de um dos caminhos que o acadêmico encontra para o desenvolvimento de uma formação acadêmica mais completa, pois os projetos integram a teoria e a prática numa relação direta de comunicação com a sociedade, possibilitando, ainda, a troca de saberes entre os acadêmicos e os participantes, e, assim, acontece a socialização e a construção de novos conhecimentos. Nos casos específicos dos cursos de licenciatura, permitem o desenvolvimento das práticas docentes e o consequente desenvolvimento de metodologias de ensino que irão potencializar a sua formação (MANCHUR; SURIANI; CUNHA, 2013).

Essa prática pode resultar em, no mínimo, duas fontes de atuação: a do próprio acadêmico, quando as mudanças acontecem no próprio indivíduo, e na comunidade, quando o acadêmico atua como intermediador dessa mudança. Esse fato pode ser visualizado, por exemplo, através de sua participação em projetos de extensão voltados para a comunidade, principalmente nos cursos da área da saúde e educação. Assim, os participantes poderão utilizar o conhecimento adquirido no dia a dia, permitindo também que transmitam essa vivência aos demais colegas universitários e que utilizem em sua vida profissional. Observamos que eles desenvolveram as atividades de tutoria com motivação e entusiasmo, atuação que pode ainda despertar neles um maior interesse pela temática sobre deficiência.

Segundo Santos e Almeida Filho (2008, p. 75), as atividades de extensão "devem ter como objetivo prioritário o apoio solidário na resolução dos problemas da exclusão e da discriminação sociais e de tal modo que nele se dê voz aos grupos excluídos e discriminados". Percebemos que oportunizar aos acadêmicos uma vivência direta com pessoas com deficiência possibilitou mudanças conceituais e atitudinais, proporcionando-lhes que passassem a enxergar com mais naturalidade as diferenças e, ao mesmo tempo, a respeitar as peculiaridades de cada indivíduo.

Portanto, a participação em projetos de extensão permite aos acadêmicos colocarem em prática os conhecimentos obtidos na teoria, e que aprendam, ainda mais, com a conexão firmada entre a universidade e a comunidade, através da participação nos projetos extensionistas, ou seja, o conhecimento beneficiando a população e fomentando a promoção da saúde. Assim, dialogar sobre a formação de futuros professores nos cursos de graduação significa, dentre outras coisas, pensar e repensar as inúmeras etapas que constituem esse processo, na busca de uma formação integral.

A relação ensino-aprendizagem é bastante dinâmica, ainda mais quando consideramos uma 
geração extremamente tecnológica, e que tem a teoria disponível, muitas vezes com um único apertar de tecla. Especificamente nos cursos de graduação em educação física, fonte de interesse deste estudo, estamos falando sobre ensinar pessoas que futuramente irão ensinar outro alguém, ou seja, ensinar sobre ensinar.

A tutoria trata-se de uma estratégia de ensino que vem sendo utilizada para diferentes finalidades, sempre objetivando a melhora no processo aprendizagem do aluno/tutorado, qual seja, encontrar tutores nos cursos ensino a distância, nos cursos superiores (e aqui se destacam os cursos das áreas da saúde) e, sobretudo, no processo de ensinoaprendizagem de alunos com deficiências, seja nos conteúdos de "sala de aula" ou nas aulas de educação física inclusiva.

Segundo Topping (2000 p. 3), "a tutoria pode ser definida como um processo em que um grupo de pessoas, não necessariamente professores, ajuda e apoia a aprendizagem de outros de uma forma interativa, significativa e sistemática". Ainda, segundo o mesmo autor, podem atuar como tutores os próprios pais dos participantes da atividade, demais adultos ou familiares, e até mesmo voluntários.

$\mathrm{Na}$ literatura, encontramos outras designações que são usadas como sinônimos de tutoria: Tutoria aos pares, Tutoria entre iguais, Aprendizagem de pares, Tutoria entre pares, Aprendizagem entre iguais ou Programas de tutoria (TOPPING, 2000). O desenvolvimento da tutoria pode acontecer de modo fixo, quando as funções entre tutor e tutorado não são recíprocas, ou pode acontecer a troca de papéis entre as pessoas envolvidas (MIRAVET; CIGES; GARCÍA, 2014).

Quando utilizada a tutoria em aulas de educação física escolar, estudos já realizados demonstram a efetividade desta estratégia para os alunos com deficiência, seja cognitiva, física ou sensorial (KLAVINA; BLOCK, 2008; QI; HA, 2012), ressaltando que os benefícios da utilização dos tutores em aulas de educação física inclusiva contribuem para uma participação mais efetiva de alunos com deficiência, além do fato de tutor e tutorado aprenderem juntos, principalmente sobre as diferenças, e, portanto, considerada uma estratégia eficiente para este fim.

Nos estudos aqui citados, os tutores que participaram das aulas aprendiam e realizavam as mesmas atividades que seus colegas com deficiência, ou seja, alunos da mesma turma. Acreditamos que a tutoria trata-se de uma estratégia que deve ser utilizada em atividades esportivas destinadas a pessoas com deficiência não somente nas aulas de educação física escolar, mas que possamos expandir esta prática para outros níveis de ensino, assim como para pessoas que participem em equipes recreativas ou de competição. Com a tutoria, o professor terá auxílio para desenvolver as atividades propostas e o alcance desta intervenção, quando consideramos o processo ensinoaprendizagem será mais amplo, através do uso de tutores treinados.

Ademais, destaca-se que, conforme apontado por Gomes Cezário et al. (2010), a articulação entre os diversos setores sociais com interesses em comum (como no caso da saúde), e a participação social, devem resultar numa melhoria nas condições e qualidade de vida de indivíduos e coletividades, especialmente relacionadas ao empoderamento dos indivíduos, ou seja, na capacidade dos cidadãos de atuarem sobre os determinantes sociais de sua própria saúde. Neste sentido, e pela possível condição de vulnerabilidade, podemos considerar o estímulo à prática da atividade física para as pessoas com deficiência uma estratégia de empoderamento para esta população.

Promover o empoderamento de pessoas com deficiência na sociedade atual é um desafio em busca de mudanças de situações biossocioculturais, e provocar esta transformação entre os que têm o poder ou não resultará numa condição de maior equidade (NASCIMENTO, 2016).

Neste sentido, a prática de uma atividade esportiva revela um mecanismo que pode e deve ser usado para este fim, pois, segundo Nascimento (2016, p. 31), "no ponto de vista da "educação empoderadora", um entre os vários mecanismos para acarretar transformações nos indivíduos é através de espaços educativos por meio do esporte". Portanto, o presente projeto de extensão propiciou também distintos benefícios para as pessoas com deficiência participantes.

\section{Conclusão}

A participação de estudantes para atuar em projetos de extensão com pessoas com deficiência se enquadra entre os aspectos fundamentais para a diminuição de atitudes preconceituosas e exclusivas, e como visualizamos nos capítulos anteriores, trata-se de um processo histórico, cujo paradigma precisa ser interrompido com urgência.

As angústias desses futuros profissionais do movimento estavam pautadas nas dificuldades de lidar com o "corpo diferente", já que o diferente tem sido, desde a era primitiva, constantemente eliminado ou excluído das mais diferentes esferas que compõem uma sociedade. A visão que se tem do diferente, ainda nos dias de hoje, gera preconceito, estigma $\mathrm{e}$ estereótipo, e isso precisa ser mudado para que a tão sonhada inclusão transforme-se em realidade. Assim, a oportunidade dos futuros professores vivenciarem essa prática e, de certa forma, enxergarem a realidade da pessoa com deficiência é, portanto, um dos caminhos possíveis em busca de uma sociedade com maior equidade. 


\section{Referências}

ABENHAIM, E. Deficiência mental, aprendizagem e desenvolvimento. In: DIAZ, F. et al. (Orgs). Educação inclusiva, deficiência e contexto social: questões contemporâneas. Salvador: EDUFBA, p. 237-243, 2009. Disponivel em: $<\mathrm{http}: / /$ books.scielo.org/id/rp6gk/22>. Acesso em 29 jun. 2017.

ARROYO, D. M. P.; ROCHA, M. S. P. M. Librandi. Metaavaliacão de uma extensão universitária: Estudo de caso. Ávaliação, Campinas, Sorocaba, v. 15, n. 2, p. 131157, Jul. 2010. Disponível em:

$<$ http://www.scielo.br/scielo.php?script=sci arttext\&pid=S1 414-40772010000200008\&lng $=$ en $\& \mathrm{nrm}=\mathrm{iso}>$. Acesso em: 25 ago. 2017.

Brasil. Decreto $n^{\circ} 6.949$, de 25 de agosto de 2009. Promulga a Convencão Internacional sobre os Direitos das Pessoas com Deficiência e seu Protocolo Facultativo, assinados em Nova York, em 30 de março de 2007. Diário Oficial [da] República Federativa do Brasil, Brasília, DF, 26 ago. 2009. Seção 1 , p. 3

DOS SANTOS, M. P. Extensão universitária: espaço de aprendizagem profissional e suas relações com o ensino e a pesquisa na educacão superior. Revista Conexão UEPG, v. 8, n. 2, p. 154-163, 2012.

FOLETTO PIVETTA, H. M. et al. Ensino, pesquisa e extensão universitária: em busca de uma integração efetiva. Linhas Críticas, v. 16, n. 31, 2010. Disponível em: $<$ http://periodicos.unb.br/index.php/linhascriticas/article/vie wFile/3028/2628>. Acesso em: 15 jun. 2017.

GOMES CEZARIO, K. et al. Promoção da saúde e deficiência visual: produção das pós-graduações brasileiras. Revista da Rede de Enfermagem do Nordeste, v. 11, n. 2, 2010.

IBGE, CENSO Demográfico 2010, Características gerais da população, religião e pessoas com deficiência, Instituto Brasileiro de Geografia e Estatística 2012 disponível em: http://biblioteca.ibge.gov.br/visualizacao/periodicos/94/cd 2 010_religiao_deficiencia.pdf. Acesso em: 16 jul. 2017.

KLAVINA A.; BLOCK, M. E. The effect of peer tutoring on interaction behaviors in inclusive physical education. Adapted physical activity quarterly, v. 25, n. 5 , p. 132158,2008

MANCHUR, J.; SURIANI, A. L. A.; DA CUNHA, M. C. A contribuição de projetos de extensão na formação profissional de graduandos de licenciaturas. Revista Conexão UEPG, v. 9, n. 2, p. 334-341, 2013.
MIRAVET, L. M.; CIGES, A. S.; GARCÍA, O. M. An experience of reciprocal peer tutoring at the university. Procedia-Social and Behavioral Sciences, v. 116 , p. 2809-2812, 2014.

Nascimento, L.C.G. Natação paralímpica de atletas com deficiência Intelectual: promoç̃o de saúde e análise cinemática. 2016. 112 f. Tese (Doutorado em Promoção da Saúde) Universidade de Franca, Franca, 2016

NUNES, A. L. P. F.; DA CRUZ SILVA, M. B. A extensão universitária no ensino superior e a sociedade. Mal-Estar e Sociedade, v. 4, n. 7, p. 119-133, 2011. Disponível em: < http://revista.uemg.br/index.php/malestar/article/view/60/89 >. Acesso em: 01 ago. 2017.

OMOTE, S. Deficiência e não deficiência: recortes do mesmo tecido. Revista Brasileira de Educação Especial, v.1, n.2, p. 65-73, 1994.

QI, J., HÁ, A.S. Inclusion in Physical Education: A review of literature. International Journal of Disability,

Development and Education, v. 59, n. 3, p. 257-281, 2012.

SANTOS, B. S.; ALMEIDA FILHO, N. A universidade no século XXI: para uma universidade nova. Almedina, 2008. Disponível em: <

http://www.boaventuradesousasantos.pt/media/A\%20Univer sidade $\% 20$ no $\% 20$ Seculo $\% 20 X X I . p d f \$$.

SARMIENTO, J. P. Healthy universities: mapping healthpromotion interventions. Health Education, v. 117, n. 2, p. 162-175, 2017. Disponível em:

$<$ http://www.emeraldinsight.com/doi/pdfplus/10.1108/HE07-2016-0028>. Acesso em: 14 jun. 2017.

TOPPING K. Tutoria Academia Internacional de Educação. UNESCO Série Práticas Educativas: Departamento Internacional de Educação, 2000.

WHO. WORLD HEALTH ORGANIZATION. International classification of impairment, disabilities, and handicaps: a manual of classification relating the consequences of disease, published in accordance with resolution WHA29. 35 of the Twenty-ninth World Health Assembly, 1976. Geneva, 1980.

World Health Organization. Relatório Mundial sobre a deficiência. São Paulo: SEDPcD, 2012.

ZANUTTO, A.; ISHIDA, S. P.; DUARTE, E. R. Projeto de extensão "Orquestrando a reciclagem". Revista Conexão UEPG, v. 13, n. 2, p. 340-349, 2017. 